\title{
A Novel Method of Interpreting the Relationship between Workload and Injury Occurrence: A Case Study
}

\author{
Case Study \\ Diarmuid O'Dowd Hill, BSc \\ diarmuid@odowdhill.com \\ ORCID: 0000-0001-6050-2916 \\ Institute of Technology Carlow
}

This Work Was Funded by the Irish Research Council Employment Based Masters Scheme: EBPPG / 2019 /40

A Novel Method of Interpreting Workload

Key Words: Youth; Rugby; Workload; ACWR; EWMA; Injuries; Load

The authors report no conflicts of interest

Abstract word count: 183

Total Word count: 1481

Number of figures: 3 


\title{
A Novel Method of Interpreting the Relationship between Workload
}

\author{
and Injury Occurrence: A Case Study
}

\section{Abstract}

Workloads may be classified as either internal or external. Internal workloads (heart rate, blood lactate, session rate of perceived exertion (SRPE) are categorised as the individual's response to an external workload (volume, intensity). SRPE is an easily monitored variable which has been shown to have possible associations with other internal $(1)$ and injury rates $(2,3)$ in contact sports. This case study describes the internal workload monitoring of a schoolboy rugby player during the entirety of his season (September to March) with the subsequent relationships between workload and time loss injuries being explored. Time loss injuries are defined as injuries which caused the individual to miss training or match play time (4). Acute to chronic workload ratio (ACWR) and exponentially weighted moving average (EWMA) were monitored, a novel ACWR:EWMA ratio was also calculated. High ACWR and low EWMA values were associated with injury occurrence, the ACWR:EWMA ratio may account for this. EWMA may be a better predictor of "time loss" injuries than ACWR. The ACWR:EWMA ratio may merit further scientific exploration in the future.

\section{Intro}

Rugby is an intermittent collision sport, in which evidence suggests that workload monitoring is associated with a participants' injury rates $(2,3,5)$. Workloads can be classified as internal 
(session rate of perceived exertion (SRPE), heart rate) or external (volume, intensity). Internal workloads may vary widely between two individuals completing the same external workload due to moderating factors such as fitness (1). Therefore, Internal workloads may give a better indication of individual fitness \& fatigue measures.

Internal workload is often calculated through the use of sRPE using Foster's borg scale (6), in which the participants rate their own exertion on a borg scale of $1-10$. This is then multiplied by the duration (minutes) of the session. Results are given in arbitrary units (AU). sRPE may have an association with physiological internal variables such as heart rate and blood lactate (1), making it a useful tool for monitoring an athletes' physiological response to a training stimulus.

This case study will focus on ACWR and EWMA; secondary to referenced strong associations with injuries in rugby union cohorts $(2,3)$. From this, a novel ACWR:EWMA ratio will be used to exhibit any possible interactions between ACWR and EWMA. Injuries will be defined as "time loss" injuries, as standardised within rugby by Fuller et al, (2007). Time loss injuries are injuries which result in the individual missing training or match play time.

The overall aim of this case study is to explore the relationship between EWMA, ACWR and the possible interaction of both with regards to "time loss" injuries over the course of a schoolboy rugby players season.

\section{Methods}




\section{Participant}

The participant was an Irish schoolboy rugby player (age; $18.2 \mathrm{yrs}$, weight; $79.5 \mathrm{~kg}$, height $172.5 \mathrm{~cm})$. The participant was in his final eligible year of playing schoolboy rugby.

\section{Data Collection}

Internal load was monitored by the participant through the use of an online form (Figure 1.1), which was shared at 8pm daily to the communication application which was used by the team. Data was collected throughout the entirety of the Connacht schoolboy rugby season (September to March).

\section{ACWR}

ACWR was calculated as the following workload ratio; acute:chronic. Acute workload was the sum of the previous seven days' workload. Chronic workload was the sum of the previous 28 days/7.

\section{EWMA}

EWMA was calculated using the following equation $=\left(\mathrm{F}^{*}\right.$ previous day workload $+(1-\mathrm{F}) *$ Sum (cumulative during period)/(1/0.1). Where $\mathrm{F}=$ the decay factor 0.1 and the cumulative load period $=$ the previous 10 days (2).

\section{ACWR: Ratio}

EWMA:ACWR ratio was calculated using the following equation $=($ ACWR/EWMA $) * 1000$. 


\section{Injury recording}

Injuries were recorded using the protocol put forward by Fuller et al, (2007) Recorded injuries recorded were defined as "time loss" injuries. "Time loss" injuries are injuries which occur during rugby training or match play and cause a loss of training or playing time (Fuller et al., 2007). Time loss injuries were recorded by the strength and conditioning coach in conjunction with guidance from the school's physiotherapist.

\section{Results}

Results were subcategorised by injury stage.

Injury stage was categorised as;

○ Not injured; any injury free period excluding the week preceding injury.

- Week preceding injury; the seven-day period preceding injury occurrence. If less than 7 days between injury periods, total days between injury periods was used.

Occurrence of injury; the day in which injury occurred.

O Injured; the period in which the athlete was excluded from training and match play due to the injury.

\section{ACWR}

No significance was found between injury occurrence $(1.3 \pm 0.5)$ and all other injury periods; not

injured $(1.1 \pm 0.4)$, week preceding injury $(1.0 \pm 0.5)$ and injured $(0.9 \pm 0.4)$. There was a moderate 
positive effect size $(d=0.5)$ between ACWR when the athlete was not injured and at injury occurrence. ACWR had a weak negative relationship to injury status $(r=-0.19)$

\section{EWMA}

EWMA was significantly lower at injury occurrence (123AU $\pm 71 \mathrm{AU})$ than when not injured (272AU士90AU, p<0.01, Cl; -259AU, -38AU). EWMA was significantly lower at the week preceding

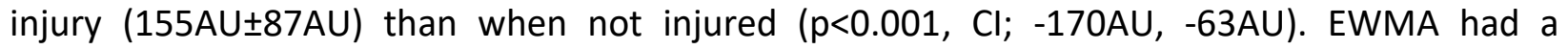
moderate negative relationship to injury status $(r=-0.53)$.

\section{ACWR:EWMA Ratio}

ACWR:EWMA ratio was significantly higher at injury occurrence (12AU $\pm 7 A U)$ than all other periods; not injured (4AU $\pm 2 A U, p<0.001 \mathrm{Cl}$; $4 A U, 12 \mathrm{AU})$, week preceding injury (7AU $\pm 5 A U$,

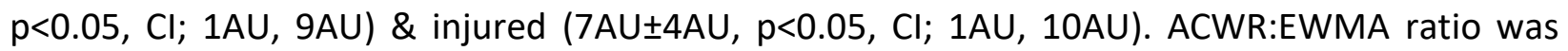
significantly higher at the week preceding injury than when not injured $(p<0.001)$. ACWR:EWMA ratio had a weak positive relationship to injury status $(r=0.32)$ 


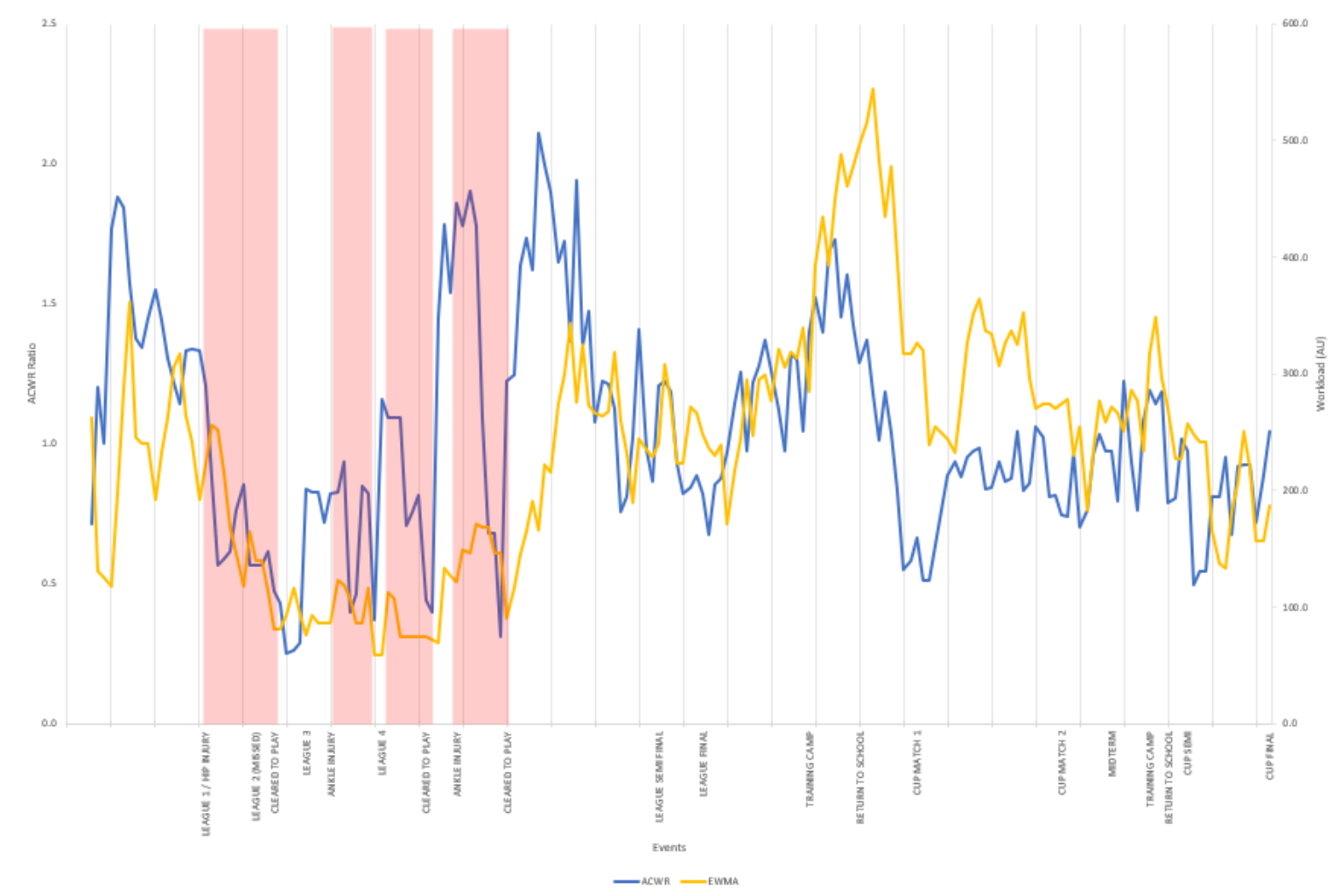

Figure 1.1: Exponentially Weighted Moving Average (EWMA) and Acute to Chronic Workload Ratio (ACWR) across the schoolboy rugby season from September to March, with key events. Weeks are indicated by vertical grid lines. Red blocks indicate periods of time loss injuries. 


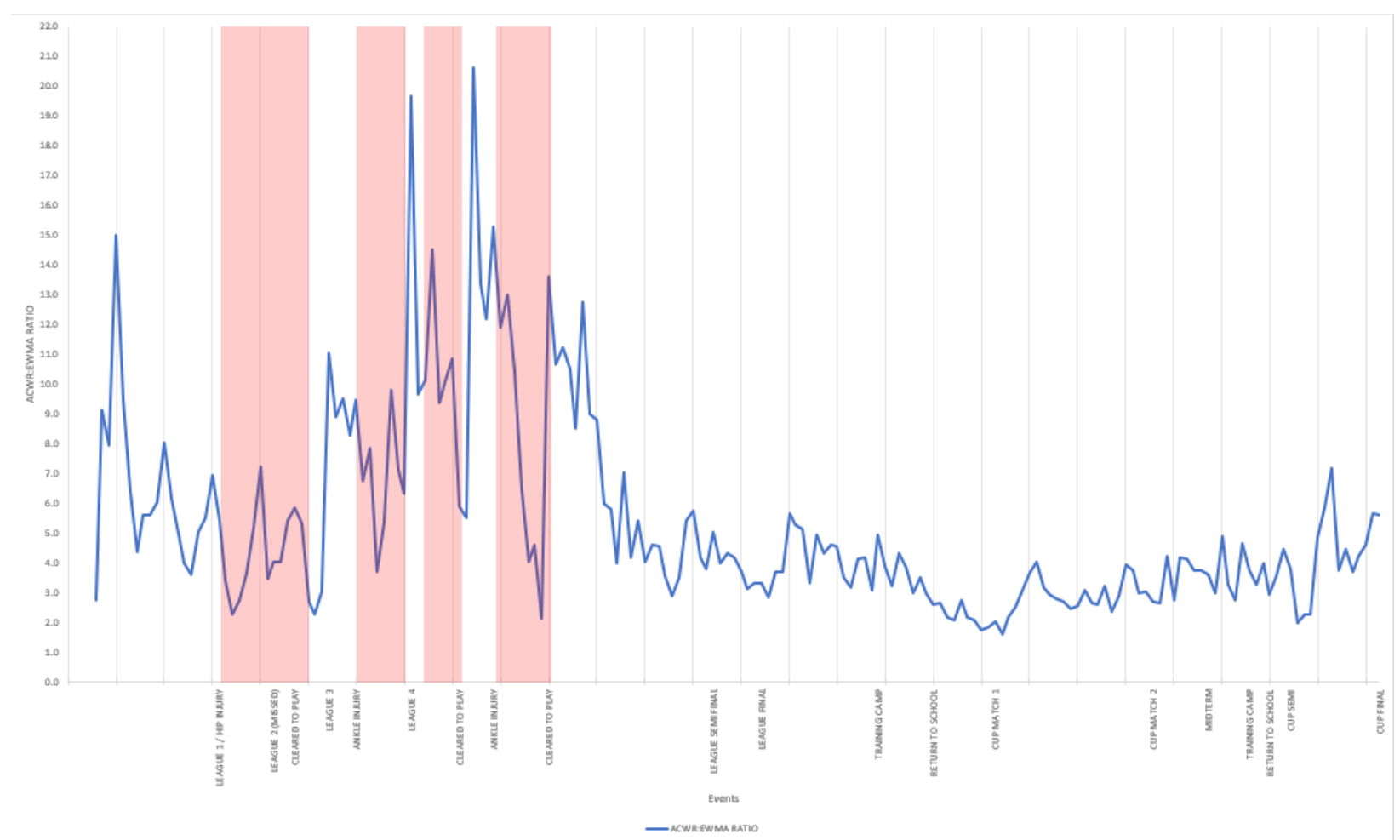

Figure 2.1: Acute to Chronic Workload Ratio (ACWR): Exponentially Weighted Moving Average (EWMA) as a ratio across the schoolboy rugby season from September to March, with key events. Weeks are indicated by vertical grid lines. Red blocks indicate periods of time loss injuries.

\section{Discussion}

Although there was no significance between ACWR when not injured and at the time of injury occurrence, the small effect size may indicate that higher ACWR values play a role in injury occurrence. This may be compounded when in conjunction with low EWMA values (Figure 2.1). This is highlighted by significantly lower EWMA value during both the week preceding injury and at time of injury occurrence. This may be due to a possible U-shaped relationship with cumulative load and injury occurrence. 
The ACWR:EWMA ratio allowed for direct comparisons across both metrics, thus accounting for both possible injury risk due to increased ACWR values and decreased EWMA values (Figure 2.1). The merit of this ratio in predicting injuries may be apparent when considering the significantly higher value at the time of injury occurrence to all other injury periods. As with EWMA, the ACWR:EWMA ratio also showed possible promise in detecting injuries in the week prior to injury occurrence.

Although these findings are in line with previous research, some limitations exist; The workload prior to the study was not accounted for and differentiation in training type is not made.

\section{Conclusions}

EWMA may be a better predictor of "time loss" injury occurrence than ACWR. The ACWR:EWMA ratio may be of use with regards to injury prevention. It is recommended that further studies be done.

\section{References}

1. Gabbett TJ. The Development and Application of an Injury Prediction Model for Noncontact, Soft-Tissue Injuries in Elite Collision Sport Athletes. Journal of Strength and Conditioning Research. 2010;24(10):2593-603.

2. Kara. Injuries in Professional Rugby Union : a Study of Five Years of Injury Data With Training Loads and Travel As Co-Variates. PhD. 2013.

3. Williams S, Trewartha G, Cross MJ, Kemp SPT, Stokes KA. Monitoring What Matters: A Systematic Process for Selecting Training-Load Measures. International Journal of Sports Physiology and Performance. 2017;12(s2):S2-101-S2-6. 
4. Fuller CW, Molloy MG, Bagate C, Bahr R, Brooks JHM, Donson H, et al. Consensus statement on injury definitions and data collection procedures for studies of injuries in rugby union. British Journal of Sports Medicine. 2007;41(5):328-31.

5. Jones CM, Griffiths PC, Mellalieu SD. Training Load and Fatigue Marker Associations with Injury and IIness: A Systematic Review of Longitudinal Studies. Sports Medicine. 2017;47(5):943-74.

6. Foster $\mathrm{C}$. Monitoring training in athletes with reference to overtraining syndrome. Medicine \& Science in Sports \& Exercise. 1998;30(7):1164-8. 